TRANSACTIONS OF THE

AMERICAN MATHEMATICAL SOCIETY

Volume 349, Number 11, November 1997, Pages 4415-4427

S $0002-9947(97) 02041-2$

\title{
APPROXIMATION BY HARMONIC FUNCTIONS
}

\author{
EVGENY A. POLETSKY
}

\begin{abstract}
For a compact set $X \subset \mathbb{R}^{n}$ we construct a restoring covering for the space $h(X)$ of real-valued functions on $X$ which can be uniformly approximated by harmonic functions. Functions from $h(X)$ restricted to an element $Y$ of this covering possess some analytic properties. In particular, every nonnegative function $f \in h(Y)$, equal to 0 on an open non-void set, is equal to 0 on $Y$. Moreover, when $n=2$, the algebra $H(Y)$ of complexvalued functions on $Y$ which can be uniformly approximated by holomorphic functions is analytic. These theorems allow us to prove that if a compact set $X \subset \mathbb{C}$ has a nontrivial Jensen measure, then $X$ contains a nontrivial compact set $Y$ with analytic algebra $H(Y)$.
\end{abstract}

\section{INTRODUCTION}

The Shilov-Bishop theorem (see [Gam], Th. 13.1) asserts that any uniform algebra $A(X)$ on a compact set $X$ has an antisymmetric restoring covering, i.e., a covering of $X$ by disjoint compacta $\left\{X_{\alpha}\right\}$ such that: 1) this partition is restoring, i.e. if $\left.f \in A\right|_{X_{\alpha}}$ for all $\alpha$, then $\left.f \in A(X) ; 2\right)$ the algebras $\left.A\right|_{X_{\alpha}}$ are antisymmetric, i.e., if $\operatorname{Im} f=0$ for $f \in A\left(X_{\alpha}\right)$, then $f$ is a constant.

For $X \subset \mathbb{C}$ and the complex uniform algebra $H(X)$ of functions on $X$ which can be uniformly approximated by holomorphic functions, for several years there were attempts to find restoring coverings of $X$ by compacta $X_{\alpha}$ with analytic algebras $H\left(X_{\alpha}\right)$. Recall that a uniform algebra is analytic if every function from this algebra, which is equal to 0 on an open non-void set, is equal to 0 everywhere. However, McKissick ([M]) gave an example of a compact set $X$ in $\mathbb{C}$ such that the algebra $H(X)$ is a normal proper subset of the algebra $C(X)$ of continuous functions on $X$ (see also [S], §27). (A uniform algebra $A$ on $X$ is normal if for every pair of disjoint sets $K_{1}$ and $K_{2}$ in $X$ there exists a function $f \in A$ such that $f\left(K_{1}\right)=0$ and $f\left(K_{2}\right)=1$.) This example disproved the existence of analytic restoring coverings for $H(X)$, because the normality of $H(X)$ implies that for a compact set $Y \subset X$ the algebra $H(Y)$ is analytic if and only if $Y$ consists of one point.

In this paper for the real space $h(X)$ of functions which can be uniformly approximated by functions harmonic on neighborhoods of $X$, we prove

Theorem 1.1. For any compact set $X \subset \mathbb{R}^{n}, n \geq 2$, there exists a covering of $X$ by disjoint subsets $Q_{\alpha}$ such that

1. $f \in h(X)$ if and only if $\left.f\right|_{\bar{Q}_{\alpha}} \in h\left(\bar{Q}_{\alpha}\right)$ and

2. every nonconstant $f \in h\left(\bar{Q}_{\alpha}\right)$ cannot attain its maximum on $Q_{\alpha}$.

Received by the editors September 10, 1995.

1991 Mathematics Subject Classification. Primary 32F05; Secondary 32E25, 32E20.

Key words and phrases. Harmonic functions, potential theory, uniform algebras.

(C)1997 American Mathematical Society 
Our approach to this theorem is very geometric, and it allows us to get many properties of the sets $Q_{\alpha}$ and the spaces $h\left(\bar{Q}_{\alpha}\right)$. In particular, criteria for the identity $h(X)=C_{\mathbb{R}}(X)$, due to Keldysh [Ga] and Khavinson [Kh], and the HartogsRosenthal theorem $[\mathrm{Ga}]$ follow from our results.

Also we are able to provide additional information on compacta $X$ with analytic algebras $H(X)$. The author's interest in this topic was raised by Wermer's example of a compact set $X$ in $\mathbb{C}^{2}$ without analytic structure [W]. Goldmann proved in [G] that $H(X)$ is analytic. This example is the cluster of some perfect sequence in $\mathbb{C}^{2}$ (see $[\mathrm{P}])$, and in this paper we show that the algebra $H(X)$ is analytic whenever $X$ is the cluster of a perfect sequence in $\mathbb{C}$.

I am grateful to T. Bagby, N. Levenberg, T. McConnell and J. Wermer for useful discussions during my work on this paper. I also wish to thank P. M. Gauthier and the referee, whose comments and corrections significantly improved the paper.

\section{Properties of harmonic MEAsures}

We will denote by $B^{n}(a, r)$ and $S^{n}(a, r)$ the ball and the sphere of radius $r$ with center $a$ lying in the real $n$-dimensional space $\mathbb{R}^{n}$. We will omit $a$ and $r$ when $a=0$ and $r=1$. We also will omit $n$ when it does not lead to misunderstanding. Let $R(z, s, t)$ be the spherical shell $\left\{x \in \mathbb{R}^{n}: s<\|x-z\|<t\right\}$ and $R(s, t)=R(0, s, t)$. A standard Lebesgue measure on sets in $\mathbb{R}^{n}$ will be denoted by $m(d z)$, and $\sigma_{n}=$ $m\left(B^{n}\right)$. The surface area measure on spheres will be denoted by $s(d z)$.

The Green function $G_{D}(x, y)$ on a domain $D$ in this paper is positive and superharmonic (sph). For the unit ball $B$ in $\mathbb{R}^{n}$ let $\Gamma(t)=G_{B}(x, 0)$, where $\|x\|=t$. Then $\Gamma(t)=-\ln t$ when $n=2$, and $\Gamma(t)=t^{2-n}-1$ when $n>2$.

Let $D$ be a bounded open set in $\mathbb{R}^{n}$. Then for every point $x \in D$ there is the harmonic measure $\mu_{x}$ supported by $\partial D$ such that for every bounded Borel function $f$ on $\partial D$ the function

$$
u(x)=\int_{\partial D} f(y) \mu_{x}(d y)
$$

is the harmonic extension of $f$ into $D$ (see [H] for details). For a closed set $E \subset \mathbb{R}^{n}$ we define $\omega(x, E, D)=\mu_{x}(E)$, where $\mu$ is the harmonic measure relative to $D \backslash E$ and $x$. It follows from the general theory (see $[\mathrm{H}])$ that $\omega(x, E, D)=\inf u(x)$, where the infimum is taken over all positive sph functions on $D$ with lower limits greater than 1 at points of $E$. It is easy to see that $\omega(z, \bar{B}(0, t), B)=\omega(z, \bar{S}(0, t), B)=$ $\Gamma(\|z\|) / \Gamma(t)$, when $\|z\| \geq t$.

The following lemma provides connections between Green functions and harmonic measures.

Lemma 2.1. Let $D$ be an open set in $B \subset \mathbb{R}^{n}$ and $0 \in D$. If

$$
u_{t}(x)=\Gamma(t) \omega(x, \bar{B}(0, t), D),
$$

then $u_{t}(x) \leq u_{s}(x)$ when $t<s$ and $\|x\|>s$, and

$$
u_{0}(x)=\lim _{t \rightarrow 0} u_{t}(x)=G_{D}(x, 0) .
$$

Proof. Since $u_{t}(x) \leq \Gamma(\|x\|)$, we see that $u_{t}(x) \leq u_{s}(x)$ when $t<s$ and $\|x\|=s$. Thus $u_{t}(x) \leq u_{s}(x)$ when $\|x\|>s$.

Since $G_{D}(x, 0) \leq \Gamma(t)$ when $\|x\|=t$, we see that $u_{t}(x) \geq G_{D}(x, 0)$ when $\|x\|>t$ and, therefore, $u_{0}(x) \geq G_{D}(x, 0)$ on $D$. The function $u_{0}(x)$ is harmonic on $D \backslash\{0\}$ 
and sph on $D$. Thus $u_{0}=c G_{D}$ for some $c>0$. But $u_{0}(x) \leq \Gamma(\|x\|)$, so $u_{0}(x) \equiv$ $G_{D}(x, 0)$.

The following theorem gives us an important estimate for Green functions.

Theorem 2.2. Let $D \subset B \subset \mathbb{R}^{n}$ be an open set and $0 \in D$. Suppose that

$$
\omega(0, \bar{B}(z, r), D)=a>0,
$$

$k>1$, and $k r \leq\|z\| / 2$. Then there is a constant $b=b(k, n)>0$ such that the measure of points $y \in R(z, r, k r)$, where

$$
G_{D}(y, 0) \geq d=\frac{a b}{2 \sigma_{n}\left(k^{n}-1\right) r^{n-2}},
$$

is greater than or equal to $\frac{a b r^{2}}{2 \Gamma(\|z\| / 2)}$.

Proof. Let us find the continuous sph function $u$ on $\mathbb{R}^{n} \backslash B$ such that $\Delta u=-m(d x)$ on the spherical shell $R(1, k), u \equiv b(k, n)$ on $\mathbb{R}^{n} \backslash B(0, k)$, and $u \equiv 0$ on $S$. Clearly on $R(1, k)$

when $n=2$ and

$$
u(x)=-\frac{1}{4}\left(\|x\|^{2}-1\right)+c \ln \|x\|
$$

$$
u(x)=-\frac{1}{2 n}\left(\|x\|^{2}-1\right)-\frac{c}{\|x\|^{n-2}}+c
$$

when $n>2$. Since, as a measure, $\Delta u$ is equal to 0 on $S(0, k)$ and $u$ is a constant outside $B(0, k)$, we conclude that the normal derivative of $u$ on $S(0, k)$ is 0 . This allows us to find $c$. Direct calculations show that

$$
u(x)=-\frac{1}{4}\left(\|x\|^{2}-1\right)+\frac{k^{2}}{2} \ln \|x\|
$$

on $R(1, k)$ when $n=2$, and

$$
b(k, 2)=\frac{1-k^{2}}{4}+\frac{k^{2} \ln k}{2} .
$$

If $n>2$ then

$$
b(k, n)=\frac{1}{n-2}\left(\frac{k^{n}-1}{n}-\frac{k^{2}-1}{2}\right)
$$

and

$$
u(x)=-\frac{1}{2 n}\left(\|x\|^{2}-1\right)-\frac{k^{n}}{n(n-2)}\left(\|x\|^{2-n}-1\right)
$$

on $R(1, k)$.

Let

$$
v(x)=r^{2} u\left(\frac{x-z}{r}\right)
$$

and $R=R(z, r, k r)$.

Let $D_{1}=D \backslash \bar{B}(z, r), g(y, x)=G_{D_{1}}(x, y)$, and let $h$ be the greatest harmonic minorant of $v$ on $D_{1}$. Then by Theorem 6.18 of [H] the function

$$
f(x)=v(x)-h(x)=\int_{R \cap D} g(x, y) m(d y) .
$$


The symmetry of the Green function implies that

$$
f(0)=\int_{R \cap D} g(y, 0) m(d y) .
$$

On the other hand, by Theorems 8.11 and 8.15 of $[\mathrm{H}]$,

$$
h(x)=\int_{\partial D_{1}} v(y) \mu_{x}(d y) \leq b r^{2} \omega\left(x, \partial D, D_{1}\right)=b r^{2}(1-\omega(x, \bar{B}(z, r), D)),
$$

where in the first equality $\mu_{x}(E)=\omega\left(x, E, D_{1}\right)$ for $E \subset \partial D_{1}$. Therefore

$$
\int_{R \cap D} g(y, 0) m(d y)=f(0)=v(0)-h(0) \geq a b r^{2} .
$$

Let $E=\{y \in R: g(y, 0) \geq d\}$. Since $g(y, 0) \leq \gamma$, where $\gamma=\Gamma(\|z\| / 2)$ and $y \in R$, and $m(R)=\sigma_{n}\left(k^{n}-1\right) r^{n}$, we see that

$$
m(E) \geq \frac{a b r^{2}-d m(R)}{\gamma-d} \geq \frac{a b r^{2}}{2 \gamma}
$$

and the theorem is proved because $G_{D}(x, y) \geq g(x, y)$.

We also will need the following trivial remark.

Lemma 2.3. Let $D$ be a bounded open set in $\mathbb{R}^{n}$ and $z_{0} \in D$. If $A \subset D$ is a closed set, $\omega\left(z_{0}, A, D\right)=b, u$ is a positive sph function on $D$ and $u \geq a$ on $A$, then $u\left(z_{0}\right) \geq a b$.

Proof. Indeed

$$
u\left(z_{0}\right) \geq a \omega\left(z_{0}, A, D\right)=a b
$$

The following lemma provides lower estimates for the area $s(E)$ of a set $E$ in $S(0, t)$ through the harmonic measure of $E$.

Lemma 2.4. Let $n>2, z_{0} \in B$, and $\left\|z_{0}\right\| \geq k>0$. If a closed set $E \subset S(0, t)$, $0<t<k / 2$, and $\omega\left(z_{0}, E, R(t, 1)\right) \geq \frac{b}{\Gamma(t)}, b>0$, then there is a constant $d=$ $d(k, b, n)$ such that $s(E) \geq d t^{n-1}$.

Proof. The inversion

$$
I(x)=\frac{t x}{\|x\|^{2}}
$$

maps $R=R(t, 1)$ onto itself and the point $z_{0}$ into the point $z_{1}$ with $\left\|z_{1}\right\|=t\left\|z_{0}\right\|^{-1}$. If a set $E \subset S(0, t)$, then

$$
\omega(z, I(E), R)=\frac{1}{\|z\|^{n-2}} \omega(I(z), E, R) .
$$

Let $P_{R}$ be the Poisson kernel on the shell $R$. The function

$$
\phi(t, m)=\inf _{x, y \in S} \frac{P_{R}(z, x)}{P_{R}(z, y)}, \quad\|z\|=\frac{t}{m},
$$

is positive and continuous on the set of positive numbers $m$ and $t$ such that $t<$ $m<1$. Let us find the limit of $\phi(t, m)$ when $t$ converges to 0 and $m$ to $m_{0}$, $0<m_{0} \leq l<1$. 
If $x \in S$ then

$$
P_{R}(z, x)=P(z, x)-\int_{S(0, t)} P(y, x) \mu_{z}(d y)
$$

where

$$
P(z, x)=\frac{1}{n \sigma_{n}} \frac{1-\|z\|^{2}}{\|z-x\|^{n}}
$$

is the Poisson kernel on the unit ball and $\mu_{z}(F)=\omega(z, F, R)$ for $F \subset \partial R$. Recalling that

$$
\omega(z, S(0, t), R(t, 1))=\frac{\|z\|^{2-n}-1}{t^{2-n}-1}=\frac{m^{n-2}-t^{n-2}}{1-t^{n-2}}
$$

and the maximal value of $P(y, x)$ on $S(0, t)$ is equal to

$$
\frac{1}{n \sigma_{n}} \frac{1-t^{2}}{(1-t)^{n}}
$$

we see that

$$
P_{R}(z, x) \geq P(z, x)\left(1-\frac{1}{P(z, x)} \frac{1-t^{2}}{n \sigma_{n}(1-t)^{n}} \frac{m^{n-2}-t^{n-2}}{1-t^{n-2}}\right) .
$$

Since $P(z, x) \rightarrow\left(n \sigma_{n}\right)^{-1}$ as $\|z\|=t / m$ goes to 0 , we get

$$
\liminf _{t \rightarrow 0, m \rightarrow m_{0}} \frac{P_{R}(z, x)}{P(z, x)} \geq 1-m_{0}^{n-2} \geq 1-l^{n-2} .
$$

We know that the ratio $P(z, x) / P(z, y)$ converges uniformly in $x$ and $y$ to 1 as $\|z\| \rightarrow 0$, and $P(x, y) \geq P_{R}(x, y)$. Therefore,

$$
\liminf _{t \rightarrow 0, m \rightarrow m_{0}} \frac{P_{R}(z, x)}{P_{R}(z, y)} \geq 1-l^{n-2}
$$

and

$$
\liminf _{t \rightarrow 0, m \rightarrow m_{0}} \phi(t, m) \geq 1-l^{n-2} .
$$

Therefore, there is a constant $c=c(k, l, n)>0$ such that on the set $K=\{(t, m)$ : $k \leq m \leq l, 2 t \leq m\}$ the function $\phi(t, m) \geq c$.

Since

$$
\frac{\Gamma\left(\left\|z_{0}\right\|\right)}{\Gamma(t)} \geq \omega\left(z_{0}, E, R(t, 1)\right) \geq \frac{b}{\Gamma(t)}
$$

there is a constant $l=l(b, n)<1$ such that $\left\|z_{0}\right\| \leq l$, and according to the assumptions of our lemma $2 t \leq k \leq\left\|z_{0}\right\|$. Hence $P_{R}\left(z_{1}, x\right) \geq c P_{R}\left(z_{1}, y\right)$. Since

$$
\omega\left(z_{1}, E^{*}, R\right)=\int_{E^{*}} P_{R}\left(z_{1}, x\right) s(d x),
$$

where $E^{*}=I(E)$, we see that

$$
\frac{\omega\left(z_{0}, E, R\right)}{\omega\left(z_{0}, S(0, t), R\right)}=\frac{\omega\left(z_{1}, E^{*}, R\right)}{\omega\left(z_{1}, S, R\right)} \leq \frac{c^{-2} s\left(E^{*}\right)}{s(S)}=\frac{t^{1-n} s(E)}{n c^{2} \sigma_{n}} .
$$

But $\omega\left(z_{0}, E, R\right) \geq b / \Gamma(t)$ and $\omega\left(z_{0}, S(0, t), R\right)=\Gamma\left(\left\|z_{0}\right\|\right) / \Gamma(t)$, so

$$
s(E) \geq \frac{n \sigma_{n} c^{2} t^{n-1} b}{\Gamma\left(z_{0}\right)} \geq d t^{n-1},
$$

where $d=n \sigma_{n} c^{2} b / \Gamma(k)$.

The following theorem is an analog of Harnack's inequality for arbitrary domains. 
Theorem 2.5. Let $D \subset B$ be an open set containing the origin, and let $a, b, k$, and $m$ be positive constants with $m \geq a$. Suppose that for some point $z_{0} \in D$ we have $\left\|z_{0}\right\|>k$ and $G_{D}\left(z_{0}, 0\right) \geq b$. Then there is a constant $c=c(a, k, m, n)>0$ with the following property: for any positive sph function $u$ on $D$ which is harmonic and less than or equal to $m$ on $D \cap B(0, k)$ and whose boundary values on $\partial D \cap B(0, k)$ are equal to 0 , we have $u\left(z_{0}\right) \geq c b$ when $u(0) \geq a$.

Proof. When $n>2$ we consider the cone $C(\alpha) \subset \mathbb{R}^{n}$, which in polar coordinates $(r, \phi)$ is described as the set of points where $\phi \leq \alpha$. Let $C(\alpha, r)=C(\alpha) \cap \bar{R}(r, k / 2)$. Using Lemma 2.4 we find $d=d(k / 2, b / 2, n)$ and take $\alpha>0$ such that $s(C(\alpha) \cap S)=$ $d$. If $B^{\prime}=B(0, k / 2)$ then the function $\psi(r)=\omega\left(0, C(\alpha, r), B^{\prime}\right)$ is a decreasing function for $0<r<k$, and $\psi(r)$ converges to 1 as $r \rightarrow 0+$. The latter statement follows from the fact that by Theorem 8.27 of $[\mathrm{H}]$ the origin is a regular boundary point for the domain $B^{\prime} \backslash C(\alpha, 0)$.

When $n=2$ we take $\psi(r)=\omega\left(0, I_{r}, B^{\prime}\right)$, where $I_{r}=[r, k / 2]$ is the interval on the real axis. In this case also $\psi(r) \rightarrow 1$ as $r \rightarrow 0+$.

Let $r=r(a, k, m, n)>0$ be the maximal number such that $r \leq k / 2$ and

$$
\frac{a \psi(r)}{4}+m(1-\psi(r)) \leq \frac{a}{2}
$$

Fix a sph function $u$ on $D$ satisfying the conditions of the theorem. Let $E \subset D$ be the set of points $x$ where $u(x) \leq a / 4$, and let $E_{t}=E \cap S(0, t)$. In the first case we assume that

$$
\omega\left(z_{0}, E_{t}, D\right)<\frac{b}{2 \Gamma(t)}
$$

for some $r \leq t<k$. Then by Lemma 2.1

$$
\omega\left(z_{0}, S(0, t) \backslash E_{t}, D\right) \geq \omega\left(z_{0}, S(0, t), D\right)-\omega\left(z_{0}, E_{t}, D\right) \geq \frac{b}{2 \Gamma(t)},
$$

and by Lemma 2.3

$$
u\left(z_{0}\right) \geq \frac{a b}{8 \Gamma(t)} \geq \frac{a b}{8 \Gamma(r)} .
$$

Thus in this case the theorem holds with $c=a / 8 \Gamma(r)$.

In the second case we assume that our previous assumption is not true, i.e.,

$$
\omega\left(z_{0}, E_{t}, D\right) \geq \frac{b}{2 \Gamma(t)}
$$

for all $r \leq t<k$. Then the sets $E_{t}$ are non-empty. By Lemma 2.4, $s\left(E_{t}\right) \geq d t^{n-1}$ when $n>2$. Let $E^{\prime}=E \cap \bar{R}(r, k / 2)$. By Corollary 2 of [BT], $\omega\left(0, E^{\prime}, B^{\prime}\right) \geq \psi(r)$ when $n>2$, and the same inequality holds for $n=2$ by the Milloux-Schmidt inequality ([N], Theorem 1, p. 107). Consider the domain $D^{\prime}=B^{\prime} \backslash E^{\prime}$ and the harmonic function $v$ on $D^{\prime}$ with boundary values equal to $a / 4$ on $E^{\prime} \cap B^{\prime}$ and to $m$ on $\partial B^{\prime} \backslash E^{\prime}$. Clearly $u \leq v$ on $D^{\prime}$ and

$$
v(0) \leq \frac{a}{4} \omega\left(0, E^{\prime}, B^{\prime}\right)+m\left(1-\omega\left(0, E^{\prime}, B^{\prime}\right)\right) \leq \frac{a}{4} \psi(r)+m(1-\psi(r)) .
$$

By inequality $(1) u(0) \leq a / 2$ and we have a contradiction, which proves that the second case cannot occur and the theorem is proved.

In the next section we will need the following fact. 
Corollary 2.6. Let $D$ be an open set in $B$ containing the origin, and let $a>0$. Fix $z_{1}, z_{2} \in D$ and let $r=\min \left\{\left\|z_{1}\right\|,\left\|z_{2}\right\|\right\}>0$. There exists a constant $c=$ $c(a, r, n)>0$ such that $G_{D}\left(z_{1}, z_{2}\right) \geq c$ if $\min \left\{G_{D}\left(0, z_{1}\right), G_{D}\left(0, z_{2}\right)\right\} \geq a$.

Proof. In Theorem 2.5 let $u(x)=G_{D}\left(x, z_{2}\right)$ and $k=r / 2$. Then $u$ is harmonic on $D \cap B(0, k)$, its boundary values are equal to 0 on $\partial D \cap B(0, k)$, and $u$ does not exceed some constant $m$ depending only on $k$ on $D \cap B(0, k)$. Since $u(0) \geq a$ and $G_{D}\left(0, z_{1}\right) \geq a$, by the previous theorem $u\left(z_{1}\right)=G_{D}\left(z_{1}, z_{2}\right) \geq c=c(a, r, n)$.

\section{HARMONIC FUNCTIONS AND RESTORING COVERINGS}

A positive Borel measure $\mu$ on a compact set $X \subset \mathbb{R}^{n}$ is called a Jensen measure with barycenter $z \in X$ if

$$
u(z) \geq \int_{X} u(x) \mu(d x)
$$

for every continuous sph function $u$ defined on a neighborhood of $X$.

Since every sph function on an open set $D$ is the limit of an increasing sequence of continuous sph functions on a set $G \subset D$, we see that subaveraging property (2) holds for all sph functions. Clearly $\mu(X)=1$ and

$$
u(z)=\int_{X} u(x) \mu(d x),
$$

when $u$ is harmonic on a neighborhood of $X$.

We denote by $J_{z}(X)$ the set of all Jensen measures on $X$ with barycenter $z$. Clearly this set is convex and weak-* compact. Let $J(X)$ be the set of all Jensen measures on $X$. This set is also weak-* compact.

We denote by $h(X)$ the real linear space of continuous functions on $X$ which can be uniformly approximated by functions harmonic in neighborhoods of $X$. We say that a continuous function $h$ on $X$ is harmonic if

$$
h(z)=\int_{X} h(x) \mu(d x)
$$

for every $z \in X$ and $\mu \in J_{z}(X)$. This definition is completely justified by the following theorem.

Theorem 3.1. A continuous function $f$ on $X$ belongs to $h(X)$ if and only if it is harmonic on $X$.

Proof. Suppose that there are harmonic functions $u_{j}$ defined on neighborhoods $D_{j}$ of $X$ and converging to $f$ uniformly on $X$. Then for every $z \in X$ and $\mu \in J_{z}(X)$ we have

$$
f(z)=\lim u_{j}(z)=\lim \int_{X} u_{j}(x) \mu(d x)=\int_{X} f(x) \mu(d x) .
$$

To prove the converse statement we extend $f$ continuously on $\mathbb{R}^{n}$ as $\widetilde{f}$. Let $D_{j}$ be the $j^{-1}$-neighborhood of $X$ and $\mu_{x}^{j}$ the harmonic measure on $D_{j}$ relative to $x$. The function

$$
u_{j}(x)=\int_{\partial D_{j}} \tilde{f}(y) \mu_{x}^{j}(d y)
$$

is harmonic on $D_{j}$. 
Suppose that there are points $z_{j} \in X$ and a $\delta>0$ such that $\left|u_{j}\left(z_{j}\right)-f\left(z_{j}\right)\right|>\delta$ for all $j$. Switching if necessary to a subsequence, we may assume that $\lim z_{j}=z$ and that the measures $\mu_{j}=\mu_{z_{j}}^{j}$ weak- $*$ converge to a measure $\mu$. If $v$ is a continuous sph function in a neighborhood of $X$, then $v$ is sph on $D_{j}$ when $j$ is sufficiently large. Therefore,

$$
v\left(z_{j}\right) \geq \int_{\partial D_{j}} v(x) \mu_{j}(d x) .
$$

Thus

$$
v(z) \geq \int_{X} v(x) \mu(d x)
$$

and $\mu \in J_{z}(X)$. Since the measures $\mu_{j}$ weak-* converge to $\mu$, the sequence of

$$
u_{j}\left(z_{j}\right)=\int_{\partial D_{j}} \widetilde{f}(y) \mu_{j}(d y)
$$

converges to

$$
\int_{X} \widetilde{f}(y) \mu(d y)=f(z) .
$$

But $f\left(z_{j}\right)$ also converges to $f(z)$, and this contradiction proves the theorem.

Let $X$ be a compact set in $\mathbb{R}^{n}$. We define the harmonic measure of a closed set $E \subset \mathbb{R}^{n}$ relative to $x \in X$ as

$$
\omega(x, E, X)=\lim _{\epsilon \rightarrow 0} \omega\left(x, E, X_{\epsilon}\right),
$$

where $X_{\epsilon}=\left\{x \in \mathbb{R}^{n}: \operatorname{dist}(x, X)<\epsilon\right\}$ is the open $\epsilon$-neighborhood of $X$.

Lemma 3.2. If $E$ is a closed set in $X$, then

$$
\omega(z, E, X)=\inf _{V} \sup _{\mu} \mu(V),
$$

where $\mu$ runs through all $J_{z}(X)$ and $V$ through all open sets containing $E$.

Proof. Let us fix some $\epsilon>0$ and take an open set $V \supset E$. Then

$$
\mu(V) \leq \int_{X} \omega\left(x, \bar{V}, X_{\epsilon}\right) \mu(d x) \leq \omega\left(z, \bar{V}, X_{\epsilon}\right)
$$

for all $\mu \in J_{z}(X)$. Hence

$$
\omega(z, E, X) \geq \inf _{V} \sup _{\mu} \mu(V) .
$$

On the other hand, for the same $V$ choose an open set $W \subset V$ containing $E$ and a sequence of positive $\epsilon_{j}$ converging to 0 such that the sequence of harmonic measures $\mu_{j}$ relative to $X_{\epsilon_{j}} \backslash \bar{W}$ and $z$ weak-* converges to some measure $\mu$. Then

$$
\mu_{j}(V) \geq \mu_{j}(\bar{W})=\omega\left(z, \bar{W}, X_{\epsilon_{j}}\right) \geq \omega(z, E, X)
$$

and $\mu(V) \geq \omega(z, E, X)$. If $u$ is a continuous sph function in a neighborhood $Z$ of $X$, then

$$
u(z) \leq \int_{\mathbb{R}^{n}} u(x) \mu_{j}(d x)
$$


when $\bar{X}_{\epsilon_{j}} \subset Z$. Since $\mu$ is the weak-* limit of $\mu_{j}$, the latter inequality holds for $\mu$ also, and this means that $\mu$ is a Jensen measure on $X$ with barycenter $z$. Thus

$$
\sup _{\mu} \mu(V) \geq \omega(z, E, X)
$$

for every open set $V \supset E$ when $\mu$ runs through all $J_{z}(X)$.

For a point $z \in X$ we consider the set

$$
I_{X}(z)=I(z)=\{x \in X: \omega(z, \bar{B}(x, r), X)>0 \text { for all } r>0\} .
$$

By Lemma 3.2 if $\mu \in J_{z}(X)$, then $\operatorname{supp} \mu \subset I(z)$. The set $I(z)$ is closed and $z \in I(z)$, so the sets $I(z)$ form a covering of $X$. The presence of points other than $z$ in $I(z)$ can be deduced from

Theorem 3.3. Let $X$ be a compact set in $\mathbb{R}^{n}$ and $z \in X$. The following statements are equivalent:

(1) $I(z)=\{z\}$.

(2) If $E_{r}=X \backslash B(z, r)$, then $\omega\left(z, E_{r}, X\right)=0$ for all $r>0$.

(3) The complement $Y$ of $X$ in $\mathbb{R}^{n}$ is nonthin at $z$.

Proof. (1) $\Rightarrow(2)$. Suppose that $\omega\left(z, E_{r}, X\right)>0$ for some $r>0$. The harmonic measure $\omega(z, A, X)$ is subadditive in $A$ and, therefore, for any finite cover of $E_{r}$ by balls $B_{j}^{1}$ of radius $r_{1}=r / 2$ with centers in $E_{r}$ the harmonic measure at $z$ of at least one ball, say $B_{1}^{1}$, is greater than 0 . Covering $B_{1}^{1}$ by balls of radius $r_{2}=r_{1} / 2$, choosing a ball with positive harmonic measure at $z$, and continuing this process at infinity, we get a sequence of points $x_{j} \in E_{r}$ such that harmonic measures at $z$ of balls of radii $r_{j}=2^{-j} r$ with centers at $x_{j}$ are positive. Clearly any limit point of this sequence is in $I(z)$, and hence $I(z) \neq\{z\}$. Thus, $\omega\left(z, E_{r}, X\right)=0$.

$(2) \Rightarrow(3)$. Suppose that $Y$ is thin at $z$. By $[\mathrm{H}$, Theorem 10.3] we can find a positive sph function $u$ on a ball $B_{1}=B(z, r)$ such that $u(x) \geq 1>u(z)=a$ for $x \in Y \cap B_{1}$. The function $v=1-u$ is subharmonic and less than 1 on $B_{1}$, negative on $Y \cap B_{1}$, and $v(z)=1-a>0$. Therefore, any positive sph function on $X_{\epsilon}$, which is greater than 1 on $E_{r / 2}$, is greater than $v$ on $X_{\epsilon}$. Hence, $\omega\left(z, E_{r}, X_{\epsilon}\right)$ and, consequently, $\omega\left(z, E_{r}, X\right)$ are greater than $1-a>0$.

$(3) \Rightarrow(2)$. Suppose that $Y$ is nonthin at $z$. Let $D_{j}, j=1,2, \ldots$, be a sequence of decreasing regular domains such that $\bigcap \bar{D}_{j}=X$. Functions $u_{j}(x)=\omega\left(x, E_{r}, D_{j}\right)$ are harmonic on $D_{j} \cap B(z, r)$ and equal to 0 on $\partial D_{j} \cap B(z, r)$. If we let $u_{j}(x)=0$ for $x \in B(z, r) \backslash \bar{D}_{j}$, then $u_{j}$ will be subharmonic on $B(z, r)$. Since the sequence of $u_{j}$ decreases, its limit $u$ exists and is a subharmonic function on $B(z, r)$. By [H, Theorem 10.3] $\lim \sup _{x \rightarrow z, x \in Y} u(x)=u(z)$. But if $x \in Y \cap B(z, r)$, then $x \in B(z, r) \backslash \bar{D}_{j}$ when $j$ is large and, therefore, $u_{j}(x)=0$. So $u(x)=0$ and $u(z)=\omega\left(z, E_{r}, X\right)=0$.

$(2) \Rightarrow(1)$. For arbitrary $x \neq z$ in $X$ find positive $t$ and $r$ such that $B(x, t) \subset$ $\mathbb{R}^{n} \backslash B(z, r)$. Then $\omega(z, \bar{B}(x, t), X)=0$ and $x \notin I(z)$. Thus $I(z)=\{z\}$.

The following theorem shows that $\{I(z)\}$ is a restoring covering for $h(X)$.

Theorem 3.4. A continuous function $f$ belongs to $h(X)$ if and only if it belongs to $h(I(z))$ for all $z \in X$.

Proof. The "only if" part of the theorem is trivial. Suppose that a continuous function $f \in h(I(z))$ for all $z \in X$. If $\mu \in J_{z}(X)$, then by Lemma $3.2 \operatorname{supp} \mu \subset I(z)$. 
If $u(x)$ is a continuous sph function defined on a neighborhood $D$ of $I(z)$, then for every set $G \subset D$ containing $I(z)$ there is a sph function $v$ on $\mathbb{R}^{n}$ such that $v \equiv u$ on $G$. To get such an extension one may assume that the open set $\mathbb{R}^{n} \backslash \bar{G}$ is regular and take a function

$$
v(x)=\min (u(x), \alpha(V(x)-\beta)),
$$

where $V(x)$ is the capacitary potential of $\bar{G}$ (see [H, p. 138]) and $\alpha, \beta$ are appropriate constants. Then

$$
\int_{X} u(x) \mu(d x)=\int_{X} v(x) \mu(d x) \leq u(z) .
$$

Thus $\mu \in J_{z}(I(z))$. Therefore,

$$
f(z)=\int_{X} f(x) \mu(d x)
$$

and by Theorem 3.1 the function $f \in h(X)$.

Green functions $G_{X_{\epsilon}}(x, y)$ decrease as $\epsilon$ decreases to 0 and, hence, converge to the function $G_{X}(x, y)$, which we will call the Green function on compacta. This function is, generally, not lower but upper semicontinuous and symmetric, i.e., $G_{X}(x, y)=G_{X}(y, x)$. The following theorem states that the properties of harmonic measures proved in Section 2 stay true when the open set $D$ is replaced by a compact set $X$. From now on, we will refer to theorems from Section 2 when we need an analogous result for compacta.

Theorem 3.5. Lemma 2.1, Theorem 2.2, and Corollary 2.6 stay true when the open set $D$ in their statements is replaced by a compact set $X \subset B$ containing the origin.

Proof. We may assume that open sets $X_{\epsilon} \subset B$. For proofs of the new versions of the listed results it is enough to note that the numbers $\omega\left(x, \bar{B}(0, t), X_{\epsilon}\right)$ and $G_{X_{\epsilon}}(x, 0)$ decrease to $\omega(x, \bar{B}(0, t), X)$ and $G_{X}(x, 0)$, respectively, as $\epsilon$ decreases to 0 .

We say that $x \sim y$ for points $x, y \in X$ if $G_{X}(x, y)>0$. Let us introduce the set $Q(z)=\left\{x \in X: G_{X}(x, z)>0\right\}$.

The following theorem describes the geometry of the sets $Q(z)$ and $I(z)$, and its first three parts plus Theorem 3.4 provide the proof for Theorem 1.1.

Theorem 3.6. Let $X$ be a compact set in $\mathbb{R}^{n}$ and $z \in X$.

(1) The set $I(z)$ is the closure of the set $Q(z)$.

(2) The relation $\sim$ is an equivalence relation.

(3) (The maximum principle) If $f \in h(X)$ and $f(y)=\sup _{x \in I(z)} f(x)$, for some $y \in Q(z)$, then $f$ is constant on $I(z)$.

(4) Either $Q(z)=\{z\}$ or $m(Q(z))>0$.

Proof. 1) We may assume that the diameter of $X$ is less than 1 . Hence some translation moves any point of $X$ into the origin leaving $X$ in $B$. If $G_{X}(x, z)=a>0$ then $G_{X}(z, x)=a$, and by Lemma $2.1 \omega(z, \bar{B}(x, t), X) \geq a / \Gamma(t)$ for all sufficiently small $t$. So $Q(z) \subset I(z)$.

If $x \in I(z)$, then by Theorem 2.2 every neighborhood of $x$ contains points $y$ where $G_{X}(y, z)>0$. So $I(z) \subset \bar{Q}(z)$. 
2) We need to prove the transitivity, which follows immediately from Corollary 2.6 .

3) By (2) $Q(z)=Q(y)$, so we may assume that $y=z$. Suppose that $x \in I(z)$ and $f(x)<f(z)$. Take a ball $B^{\prime}=B(x, r)$ such that $f(y)<f(z)$ when $y \in X \cap B^{\prime}$. Since $\omega\left(z, B^{\prime}, X\right)>0$, by Lemma 3.2 there is a measure $\mu \in J_{z}(X)$ such that $\mu\left(B^{\prime}\right)>0$. Then

$$
f(z)=\int_{X} f(y) \mu(d y)<f(z),
$$

and this contradiction proves part 3$)$.

4) If $Q(z)$ contains more than one point, then by Lemma 2.1 and Theorem 2.2 every neighborhood of these points has positive measure.

Theorem 3.4 and the first and fourth part of the previous theorem yield the Hartogs-Rosenthal theorem.

Theorem 3.7. If the measure of a compact set $X \subset \mathbb{R}^{n}$ is zero, then $h(X)=$ $C_{\mathbb{R}}(X)$.

The theorem above provides only a sufficient criterion for $h(X)=C_{\mathbb{R}}(X)$. The following theorem does it in the "necessary and sufficient" form. Statements (3) and (4) are due, respectively, to M. V. Keldysh [Ga] and D. Khavinson [Kh].

Theorem 3.8. For a compact set $X \subset \mathbb{R}^{n}$ the following statements are equivalent:

(1) $h(X)=C_{\mathbb{R}}(X)$;

(2) $I(z)=\{z\}$ for all $z \in X$;

(3) $\mathbb{R}^{n} \backslash X$ is nonthin at all points of $X$;

(4) $f(x)=\|x\|^{2}$ is in $h(X)$.

Proof. (1) and (2) are equivalent by Theorem 3.4. (2) and (3) are equivalent by Theorem 3.3. Clearly (1) implies (4). If (4) is true, then $-\|x-z\|^{2} \in h(X)$ for all $z \in X$. Hence by the third part of Theorem $3.6 Q(z)=\{z\}$. So $I(z)=\{z\}$ and (1) is also true.

Definition 3.9. A compact set $X \subset \mathbb{R}^{n}$ is called a Jensen set if $X=I(z)$ for some point $z \in X$.

The simplest example of a Jensen set is the closed unit ball in $\mathbb{R}^{n}$. Also, if $Y$ is a compact set, $z \in Y$, and $X=I(z)$, then $X$ is a Jensen set. Indeed, $J_{z}(X)=J_{z}(Y)$ because all measures from $J_{z}(Y)$ are supported by $X$. Hence by Lemma $3.2 \omega(z, E, X)=\omega(z, E, Y)$ for all closed sets $E \subset X$. This means that $I_{X}(z)=I_{Y}(z)=X$.

The following example provides a non-trivial Jensen set whose interior is empty.

Example 3.10. Take, in $B$, a countable dense set $A=\left\{z_{1}, z_{2}, \ldots\right\}$ which does not contain the origin and a point $x \notin A$ with $\|x\|>1 / 2$. Let $B_{j}=B\left(x, 1 / 2^{j}\right)$, $j=1,2, \ldots$ We choose $t_{1}>0$ such that $x \notin B\left(z_{1}, t_{1}\right)$ and $\omega\left(0, \bar{B}_{1}, \bar{D}_{1}\right)>$ $\omega\left(0, \bar{B}_{1}, \bar{B}\right) / 2$, where $D_{1}=\bar{B} \backslash B\left(z_{1}, t_{1}\right)$. If $t_{1}, \ldots, t_{n-1}$ have been chosen, choose $t_{n}>0$ such that $x \notin B\left(z_{n}, t_{n}\right)$ and $\omega\left(0, \bar{B}_{j}, \bar{D}_{n}\right)>\omega\left(0, \bar{B}_{j}, D_{j}\right) / 2$, where $j=$ $1, \ldots, n$ and $D_{j}=D_{j-1} \backslash B\left(z_{j}, t_{j}\right)$. Let $Y=\bigcap D_{j}$. Then $x \in Y$. For every $\epsilon>0$ the compact set $D_{j} \subset Y_{\epsilon}$ when $j$ is sufficiently large and, therefore, $\omega\left(0, B_{j}, Y\right)>0$ for all $j$. Thus $x \in I_{Y}(0)$. Take $X=I_{Y}(0)$. The interior of $X$ is empty and $X$ is Jensen. 
Now we are able to establish analytic properties of elements of a restoring covering. These elements are Jensen sets. From the maximum principle we get

Corollary 3.11. If $X$ is a Jensen set in $\mathbb{R}^{n}$, then every non-negative function $f \in h(X)$ equal to 0 on an open non-void set is equal to 0.

As the following example shows, a point $w$ in $I(z) \backslash Q(z)$ can have a non-trivial set $Q(w)$ when $X \subset \mathbb{R}^{n}, n \geq 3$.

Example 3.12. Let $\phi(x)$ be a positive continuous function on $(-\infty, 0)$ decreasing so fast that for the set

$$
E=\bar{B}(0,1) \backslash\left\{x<0, y^{2}+z^{2}<\phi(x)\right\}
$$

in $\mathbb{R}^{3}=(x, y, z)$ the origin is an irregular point. Let

$$
F=\bar{B}(0,1) \cap\left\{x<0, y^{2}+z^{2} \leq \frac{\phi(x)}{2}\right\}
$$

and $X=E \cup F$. If $w_{1}=(-1 / 2,0,0)$ and $w_{2}=(1 / 2,0,0)$, then $0 \in I\left(w_{1}\right) \backslash Q\left(w_{1}\right)$, but $G_{X}\left(0, w_{2}\right)>0$, so $w_{2} \in I(0)$.

However for $n=2$ we have

Theorem 3.13. If $X$ is a compact set in $\mathbb{C}, z \in X$ and $w \in I(z)$, then either $Q(z)=Q(w)$ or $Q(w)=\{w\}$.

The proof of this theorem follows immediately from

Lemma 3.14. Suppose that $z$ is a point of a compact set $X \subset \mathbb{C}, w \in I(z)$, $y \notin Q(z), y \neq w$. Then $G_{X}(w, y)=0$.

Proof. If $Q(z) \cap S(w, t)=\emptyset$ for some $t<r=\|z-w\|$, then $G_{X}(x, z) \equiv 0$ on $B(w, t)$ and, hence, $w \notin I(z)$. So $Q(z) \cap S(w, t) \neq \emptyset$ for $0<t<r$. Let $s=\min \{r,\|w-y\| / 2\}$ and $E=Q(z) \cap B(w, s)$.

Let $G_{\epsilon}$ be the Green function on $X_{\epsilon}$. We fix $\delta>0$ and for every $\epsilon>0$ we consider the closure $F_{\epsilon}$ of the set

$$
\left\{x \in B(w, s) \cap X_{\epsilon}: G_{\epsilon}(x, y) \leq \delta\right\} .
$$

Since the union of $F_{\epsilon}, \epsilon>0$, contains $E$, by the Milloux-Schmidt inequality,

$$
\lim _{\epsilon \rightarrow 0} \omega\left(w, F_{\epsilon}, B(w, s) \cap X_{\epsilon}\right)=1 \text {. }
$$

So $\omega\left(w, F_{\epsilon}, B(w, s) \cap X_{\epsilon}\right)>1-\delta$ for some $\epsilon>0$, and since $G_{\epsilon}(x, y)$ is less than some constant $c$ on $B(w, s)$, we see that $G_{\epsilon}(w, y) \leq \delta(1-\delta)+c \delta$. Thus $G_{X}(w, y) \leq$ $\delta(1-\delta)+c \delta$ for any $\delta>0$, and $G_{X}(w, y)=0$.

Definition 3.15. A Jensen set $X$ is called a Wermer set if for every $z \in X$ either $I(z)=X$ or $I(z)=\{z\}$.

The previous theorem implies

Corollary 3.16. Every Jensen set $X \subset \mathbb{C}$ is a Wermer set.

Definition 3.17. A uniform algebra $A$ on a compact set $X$ is analytic if every function $f \in A$ equal to 0 on an open non-void set in $X$ is equal to 0 .

If $X$ is a compact set in $\mathbb{C}$, we denote by $H(X)$ the complex uniform algebra of continuous functions on $X$ which can be uniformly approximated on $X$ by functions holomorphic in neighborhoods of $X$.

The following theorem is a simple consequence of the Two Constants Theorem. 
Theorem 3.18. If $X$ is a Jensen set in $\mathbb{C}$, then $H(X)$ is analytic.

Proof. Let $h \in H(X)$ be equal to 0 on a non-void open set $V \subset X$. We may assume that $X=I(z)$ and $z \in V$. There is a sequence of open sets $U_{j} \supset X$ and holomorphic functions $h_{j}$ on $U_{j}$ such that $h_{j}$ converge uniformly to $h$ on $X$. We may assume that $\left|h_{j}\right| \leq 1$ on $U_{j}$. If $w \in Q(z)$ then

$$
\omega\left(w, \bar{V}, U_{j}\right) \geq \omega(w, \bar{V}, X)=a>0 .
$$

Let $b_{j}$ be the supremum of $\left|h_{j}\right|$ on $\bar{V}$. By the Two Constants Theorem $\left|h_{j}(w)\right| \leq b_{j}^{a}$, and therefore $h(w)=0$ for $w \in Q(z)$. Since $Q(z)$ is dense in $X, h \equiv 0$.

This result above yields the following:

Corollary 3.19. If a compact set $X$ in $\mathbb{C}$ has a nontrivial Jensen measure (not equal to $\delta_{z}$ ), then $X$ contains a nontrivial compact set $Y$ with analytic algebra $H(Y)$.

Proof. Let $z$ be the barycenter of a nontrivial Jensen measure on $X$. Then $Y=I(z)$ is a nontrivial Jensen set.

It follows from $[\mathrm{P}]$ that a compact set $X \subset \mathbb{C}^{n}$ is Jensen (with respect to $z$ ) if and only if $X$ is the cluster of a perfect sequence centered at $z$. Combining this result with Theorem 3.18, we get the following:

Corollary 3.20. If a compact set $X \subset \mathbb{C}$ is the cluster of a perfect sequence of mappings of the unit disk into $\mathbb{C}$, then $H(X)$ is analytic.

In $\mathbb{C}^{n}$ the situation is more complicated. The Wermer example $X$ is the cluster of a perfect sequence with analytic algebra $H(X)$. This follows from the proof of analyticity of $H(X)$ in [G]. But the compact set $Y=Y_{1} \cup Y_{2}$, where $Y_{1}=\{(z, w) \in$ $\left.\mathbb{C}^{2}:|w| \leq 1,|z|=1\right\}$ and $Y_{2}=\left\{(z, w) \in \mathbb{C}^{2}: w=0,|z| \leq 1\right\}$ is also the cluster of a perfect sequence (see $[\mathrm{P}])$. However, the algebra $H(Y)$ is not analytic because $f(z, w)=w$ is equal to 0 on $Y_{2}$.

\section{REFERENCES}

[BT] A. Baernstein II, B. A. Taylor, Spherical rearrangements, subharmonic functions, and *-functions in n-space, Duke Math. J., 43, (1976), pp. 245-268. MR 53:5906

[Gam] T. Gamelin, Uniform Algebras, Prentice-Hall, Inc., 1984. MR 53:14137 (1st ed.)

[Ga] P. M. Gauthier, Uniform approximation, In: Complex Potential Theory, Kluwer Acad. Publ., 1994, pp. 235-271. MR 96d:30048

[G] H. Goldmann, An analytic algebra without analytic structure in the spectrum, Ark. Mat., 27 (1989), 89-95. MR 90e: 46042

[H] L. L. Helms, Introduction to Potential Theory, Wiley-Interscience, 1969. MR 41:5638

[Kh] D. Khavinson, On uniform approximation by harmonic functions, Mich. Math. J., 34 (1987), 465-473. MR 89a:14026

[M] R. McKissick, A nontrivial normal sup algebra, Bull. Amer. Math. Soc., 69 (1963), 391395. MR 26:4166

[N] R. Nevanlinna, Analytic functions, Springer-Verlag, 1970. MR 43:5003

[P] E. A. Poletsky, Analytic geometry on compacta in $\mathbb{C}^{n}$, Math. Z., 222 (1996), 407-424. MR 97e:32015

[S] E. L. Stout. The Theory of Uniform Algebras, Bogden \& Quigley, Inc., Tarrytown-onHudson, NY, 1971. MR 54:11066

[W] J. Wermer, Polynomially convex hulls and analyticity, Ark. Mat., 20 (1982), 129-135. MR 84b:32021

Department of Mathematics, 215 Carnegie Hall, Syracuse University, Syracuse, New YORK 13244 